University of Nebraska - Lincoln

DigitalCommons@University of Nebraska - Lincoln

Civil Engineering Faculty Publications

Civil Engineering

June 2006

Self-similarity of Mean Flow in Pipe Turbulence

Junke Guo

University of Nebraska - Lincoln, jguo2@unl.edu

Follow this and additional works at: https://digitalcommons.unl.edu/civilengfacpub

Part of the Civil Engineering Commons

Guo, Junke, "Self-similarity of Mean Flow in Pipe Turbulence" (2006). Civil Engineering Faculty Publications. 2.

https://digitalcommons.unl.edu/civilengfacpub/2

This Article is brought to you for free and open access by the Civil Engineering at DigitalCommons@University of Nebraska - Lincoln. It has been accepted for inclusion in Civil Engineering Faculty Publications by an authorized administrator of DigitalCommons@University of Nebraska - Lincoln. 


\title{
Self-Similarity of Mean-Flow in Pipe Turbulence
}

\author{
Junke Guo* \\ University of Nebraska, Omaha, NE 68182-0178, U.S.
}

\begin{abstract}
Based on our previous modified log-wake law in turbulent pipe flows, we invent two compound similarity numbers $(Y, U)$, where $Y$ is a combination of the inner variable $y^{+}$and outer variable $\xi$, and $U$ is the pure effect of the wall. The two similarity numbers can well collapse mean velocity profile data with different moderate and large Reynolds numbers into a single universal profile. We then propose an arctangent law for the buffer layer and a general log law for the outer region in terms of $(Y, U)$. From Milikan's maximum velocity law and the Princeton superpipe data, we derive the von Kármán constant $\kappa=0.43$ and the additive constant $B \approx 6$. Using an asymptotic matching method, we obtain a self-similarity law that describes the mean velocity profile from the wall to axis; and embeds the linear law in the viscous sublayer, the quartic law in the bursting sublayer, the classic log law in the overlap, the sine-square wake law in the wake layer, and the parabolic law near the pipe axis. The proposed arctangent law, the general log law and the self-similarity law have been confirmed with the high-quality data sets, with different Reynolds numbers, including those from the Princeton superpipe, Loulou et al., Durst et al., Perry et al., and den Toonder and Nieuwstadt. Finally, as an application of the proposed laws, we improve the McKeon et al. method for Pitot probe displacement correction, which can be used to correct the widely used Zagarola and Smits data set.
\end{abstract}

\section{Nomenclature}

$A=$ constant in the arctangent law, Eq. (19)

$B=$ additive constant in the classic log law, Eq. (9)

$B_{1}=$ additive constant in Millikan's log law of the maximum velocity, Eq. (10)

$C=$ model constant in the self-similarity law, Eq. (26)

$D_{p}=$ diameter of Pitot probe in Eq. (30)

$f \quad=$ generic function

$k_{i}=$ coefficients in the arctangent law, Eq. (19), where $i=1,2,3, \cdots$

$U=$ similarity number representing the pure effect of the wall, Eq. (14e)

$u=$ mean velocity at distance $y$ from the wall

$u_{\max }=$ maximum velocity at the pipe center

$u_{\max }^{+}=$dimensionless maximum velocity, $u_{\max }^{+}=u_{\max } / u_{*}$

$u^{+}=$dimensionless velocity scaled by the shear velocity, $u^{+}=u / u_{*}$

$u_{*} \quad=$ shear velocity

$R=$ radius of pipe

$R e=$ bulk Reynolds number

$R e_{*}=$ Kármán number

$Y=$ similarity number combining the inner and outer variables, Eq. (14d)

$y=$ distance from the wall

$y_{c}=$ true velocity at the center of the probe in Eq. (31)

$y^{+}=$inner variable, $y^{+}=y u_{\star} / \nu$

$\alpha=$ dimensionless velocity gradient, Eq. (31)

$\gamma=$ Österlund diagnostic function, Eq. (15)

$\Delta y=$ correction in position due to streamline displacement in Eq. (30)

*Assistant Professor, Department of Civil Engineering, PKI 204D, 1110 S 67th St., jguo2@unl.edu. Member AIAA. 


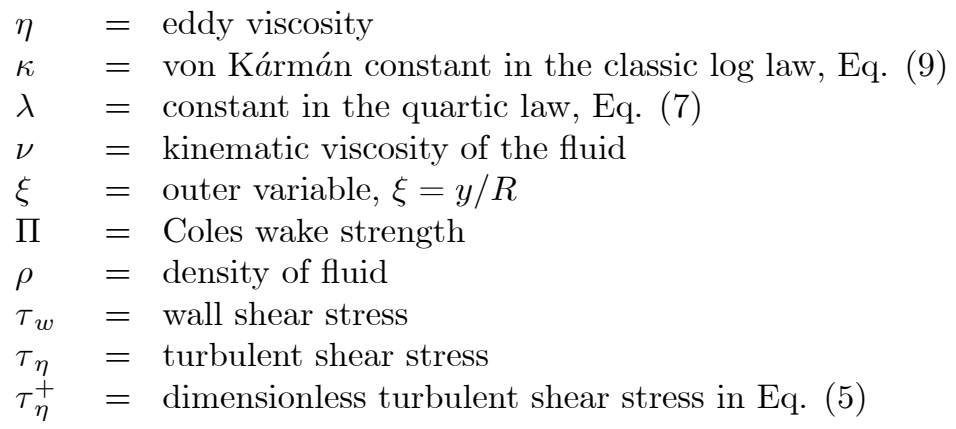

\section{Introduction}

DIPE turbulence has been studied over a century since Reynolds landmark experiment in 1883, but it is still a great unsolved problem in classical physics (Nelkin 1992, Gad-el-Hak 1997). This is because the Reynolds averaged governing equations always have more unknowns (statistical quantities) than equations; and direct numerical simulation (DNS) of the Navier-Stokes equations for large Reynolds number is always beyond the current computer capability. Therefore, dimensional reasoning, similarity and asymptotic analysis become important tools in turbulence research (Gad-el-Hak 1997).

The purpose of dimensional reasoning, similarity and asymptotic analysis is to find a self-similairity solution that is to find two similarity numbers so that they can collapse experimental velocity profiles with different Reynolds numbers from different facilities to a single universal profile. This procedure usually starts with dimensional reasoning that gives

$$
\frac{u}{u_{*}}=f\left(\frac{y u_{*}}{\nu}, \frac{y}{R}\right)
$$

where $u=$ mean velocity at distance $y$ from the wall, $u_{*}=$ shear velocity, $\nu=$ kinematic viscosity of the fluid, and $R=$ pipe radius. Equation (1a) is often rewritten as

$$
\frac{u}{u_{*}}=f\left(\frac{y u_{*}}{\nu}, \mathrm{Re}_{*}\right)
$$

where $\operatorname{Re}_{*} \equiv R u_{*} / \nu$ is the Kármán number that expresses the ratio of the outer length scale $R$ to inner length scale $\nu / u_{*}$, or

$$
\frac{u}{u_{*}}=f\left(\frac{y u_{*}}{\nu}, \operatorname{Re}\right)
$$

where Re is the bulk Reynolds number and a definition of friction factor has been applied. Equations (1a)-(1c) are still complicated because of the two independents.

Our question in this paper is: Can we find two similarity numbers that, including the variables in Eqs. (1a)-(1c), can collapse experimental velocity profiles with different Reynolds numbers from different facilities to a single universal profile? To answer this question, let us start with the following governing equation.

$$
\frac{1}{\operatorname{Re}_{*}} \frac{d u^{+}}{d \xi}+\frac{\eta}{\rho u_{*} R} \frac{d u^{+}}{d \xi}=1-\xi
$$

Inner region $(\xi \rightarrow 0)$

viscous sublayer

bursting sublayer

buffer layer

quasi-inertia layer

inertia layer $\left(y^{+} \rightarrow \infty\right)$

Outer region $\left(\operatorname{Re}_{*} \rightarrow \infty\right)$

inertia layer $(\xi \rightarrow 0)$

wake layer

near the axis

$\begin{array}{rlrl}\checkmark & \eta & \rightarrow 0 \\ \checkmark & \tau_{\eta} & \rightarrow y^{+3} \\ \checkmark & \eta & \sim \mu \\ \checkmark & \eta & >\mu \\ \mu<<\eta & \tau_{\eta} & \sim \tau_{w} \\ \operatorname{Re}_{*} & \rightarrow \infty & \tau_{\eta} & \sim \tau_{w} \\ \operatorname{Re}_{*} & \rightarrow \infty & \checkmark \\ \operatorname{Re}_{*} & \rightarrow \infty & d u^{+} / d \xi \rightarrow 0\end{array}$

$\begin{array}{lcl}\checkmark & \xi \rightarrow 0 & \text { linear law, Eq. (4) } \\ \checkmark & \xi \rightarrow 0 & \text { quartic law, Eq. (7) } \\ \checkmark & \xi \rightarrow 0 & \text { arctangent law, Eq. } \\ \checkmark & \xi \rightarrow 0 & \\ \checkmark & \xi \rightarrow 0 & \text { log law, Eq. (9) } \\ \checkmark & \xi \rightarrow 0 & \text { log law, Eq. (9) } \\ \checkmark & \checkmark & \text { log-wake law, Eq. (11 } \\ \checkmark & \checkmark & \text { parabolic law, Eq. }\end{array}$

Velocity Asymptote 
In the above $u^{+} \equiv u / u_{*}, \xi \equiv y / R, \eta=$ eddy viscosity, and $\rho=$ density of the fluid; the first term on the left-hand-side is the viscous shear, and the second the turbulent shear; the first term on the right-hand-side is the wall shear, and the second the pressure-gradient. We conventionally divide the flow domain into the inner and outer regions. The inner region is defined near the wall where $\xi \rightarrow 0$. Considering $\operatorname{Re}_{*} \xi=y u_{*} / \nu \equiv y^{+}$ in Eq. (2), the inner region solution has the functional form,

$$
u^{+}=f\left(y^{+}\right)
$$

which is called the law of the wall (Schlichting 1979, p.605).

Unlike previous studies, we divide the inner region into five layers: the viscous sublayer, bursting sublayer, buffer layer, quasi-inertia layer, and inertia layer, shown below Eq. (2). In the viscous sublayer, say $y^{+}<5$, turbulence is restricted by the wall, the eddy viscosity $\eta \rightarrow 0$. Equation (2), with the no-slip condition, gives the linear law,

$$
u^{+}=y^{+}
$$

This equation is the first asymptote for Eq. (3).

The sweep-ejection bursting process generates turbulence near the wall (Kline et al. 1967, Kim et al. 1971, Grass 1971, Grass et al. 1991). To reflect this important phenomenon in the inner region, we term the corresponding zone the bursting sublayer. From the mass conservation, Chapman and Kuhn (1986) showed that the dimensionless turbulent shear, $\tau_{\eta}^{+}$, in this layer must follow a cubic law,

$$
\tau_{\eta}^{+}=\lambda y^{+3}+\cdots
$$

where $\lambda$ is determined experimentally. Equation (2) then becomes

$$
\frac{d u^{+}}{d y^{+}}+\lambda y^{+3}+\cdots=1
$$

which results in the following quartic law for the mean velocity,

$$
u^{+}=y^{+}-\frac{\lambda}{4} y^{+4}+\cdots
$$

The value of $\lambda$ is reported about $(4-8) \times 10^{-4}$ in literature (Buschmann and Gad-el-Hak 2005). Using the Loulou et al. (1997a, b) DNS data near the wall, we obtain

$$
\lambda=\frac{1}{1150}=8.7 \times 10^{-4}
$$

which is valid till about $y^{+}=8$, shown in Fig. 1. Thus, the bursting sublayer is defined between $y^{+}=5$ and 8 , which is very thin but important in turbulence production and modeling. The quartic law of Eq. (7) is the second asymptote for Eq. (3).

We define the buffer layer between $y^{+}=8$ and 30 where the viscous and turbulent shears are comparable. Spalding's law of the wall (White 1991, p.415) is often used to describe this layer. Except for its implicit form, McKeon et al. (2003) showed that it cannot well approximate the new refined experimental data. The other popular one is van Driest's law of the wall (Schliching 1979, p.604). For example, Guo et al. (2005) combined it with the modified log-wake law to describe the entire turbulent boundary layer flows. But it cannot reflect the cubic turbulent shear or the quartic velocity profile in the bursting sublayer (Buschmann and Gad-el-Hak 2005). We will propose an arctangent law for this layer later.

Recently the Princeton superpipe experiments (Zagarola and Smits 1998, McKeon et al. 2004) revealed that between the buffer and inertia layers, a power law layer exists, where the turbulent shear prevails but the viscous shear cannot be neglected. We term the corresponding zone a quasi-inertia layer. Like the buffer layer, we cannot derive an analytic asymptote in this layer; the power law is simply empirical.

The inertia layer has been well established where $y^{+} \rightarrow \infty$ and the viscous effect can be completely neglected. In the 1930s Prandtl and von Kármán (Schlichting 1979, Chaps. 29 and 30) reasoned that Eq. (3) in this layer can be expressed by the log law,

$$
u^{+}=\frac{1}{\kappa} \ln y^{+}+B
$$




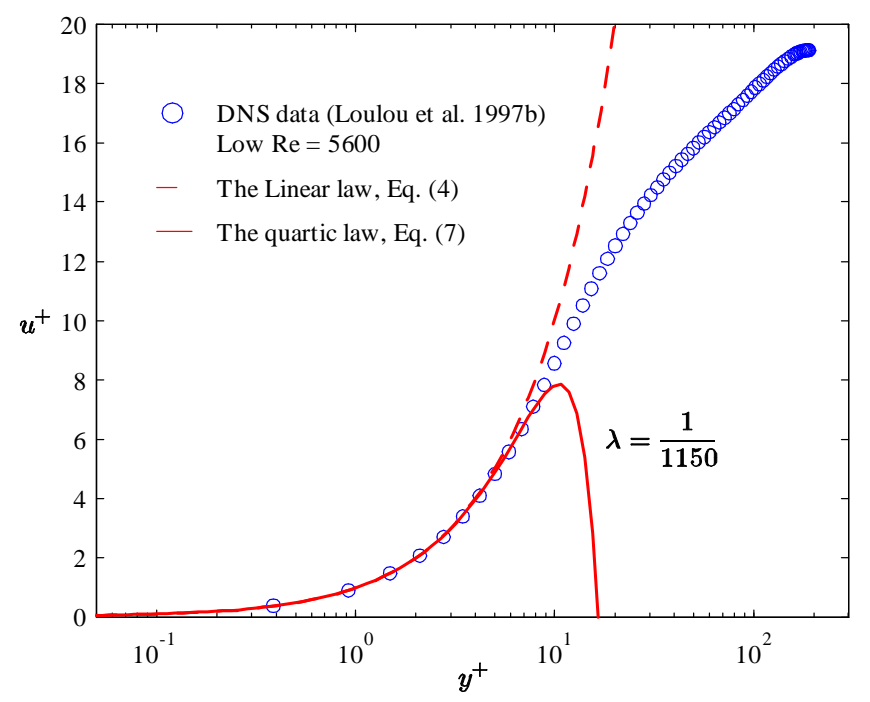

Figure 1. Check of the linear and quartic asymptotes with data of low Re turbulence where the laminar and bursting sublayers are amplified.

where $\kappa=$ von Kármán constant, and $B=$ additive constant. From Eq. (2) we can see that there is an overlap when $y^{+} \rightarrow \infty$ in the inner region and $\xi \rightarrow 0$ in the outer region. Milikan (1938), applying asymptotic matching, proved that Eq. (3) must be the logarithmic function above. The difficulty in applying Eq. (9) is that there does not exist an objective method to determine the overlap range, which causes the different values of $\kappa$ and $B$ in literature (Zanoun et al. 2003). In fact, while deriving Eq. (9), Milikan simultaneously obtained that the maximum velocity $u_{\max }$ must also follow a log law (Panton 2005),

$$
u_{\max }^{+}=\frac{1}{\kappa} \ln \operatorname{Re}_{*}+B_{1}
$$

where $u_{\max }^{+} \equiv u_{\max } / u_{*}$, the von Kármán constant $\kappa$ is the same as that in Eq. (9), and $B_{1}$ is another constant. The importance of Eq. (10), which is often overlooked in literature, is that it provides an objective way to determine the von Kármán constant $\kappa$ where we do not need to concern the range of the overlap. Equation (9) is our third asymptote for Eq. (3).

Including the overlap, the outer region is also called the core region. The prerequisite for this region is $\operatorname{Re}_{*} \rightarrow \infty$, shown below Eq. (2); otherwise, an outer region does not exist at all. Since the log law of Eq. (9) is a common term between the inner and outer regions, Coles (1956) and Hinze (1975, p.698) extended the $\log$ law beyond the overlap by adding the law of the wake, i.e.

$$
u^{+}=\underbrace{\left(\frac{1}{\kappa} \ln y^{+}+B\right)}_{\text {the log law }}+\underbrace{\frac{2 \Pi}{\kappa} \sin ^{2} \frac{\pi \xi}{2}}_{\text {the wake law }}
$$

where $\Pi=$ wake strength. Equation (11) is in the form of Eq. (1a). Experiments (Hinze 1975, p.699) showed that the log-wake law above is a good approximation till about $90 \%$ of the radius from the wall, $\xi \leq 0.9$; but it is not valid near the axis since it does not meet the axisymmetric requirement, $d u / d y=0$ at $\xi=1$. Equation (11) is a widely accepted experimental fact in the wake layer.

The last layer is the zone near the axis where the axisymmetric requirement results in the parabolic defect law below,

$$
u_{\max }^{+}-u^{+} \propto(1-\xi)^{2}
$$

which can be derived from a Taylor series expansion at $\xi=1$ (Guo and Julien 2003). To force the log-wake law of Eq. (11) to meet the above parabolic asymptote near the axis, Guo and Julien added a correction term to Eq. (11),

$$
u^{+}=\left(\frac{1}{\kappa} \ln y^{+}+B\right)+\frac{2 \Pi}{\kappa} \sin ^{2} \frac{\pi \xi}{2}-\frac{\xi^{3}}{3 \kappa}
$$


The cubic term corrects the log law gradient to be zero at the axis and is intrinsically a part of the effect of the wall. Guo and Julien pointed out that the sine-square function in Eq. (13) reflects the pressure-gradient effects and the wake strength $\Pi$ is about the same as the von Kármán constant, $\Pi \approx \kappa$, for large Reynolds number. Equation (13) has been verified with the Zagarola and Smits superpipe data set in the outer region. We will take it as our preliminary analysis to formulate the hypotheses in this paper.

In summary, we have four asymptotes, Eqs. (4), (7), (9) and (12), which result from the boundary conditions, asymptotic matching and mass conservation. Any reasonable pipe turbulence model should embed these four asymptotes. In addition, if possible, it should contain the sine-square wake law. The modified log-wake law of Eq. (13) has included Eqs. (9) and (12) as well as the sine-square wake law.

Although the Barenblatt power law (Barenblatt 1993; Barenblatt et al. 1997a, 1997b) caused a stir on wall turbulence research in the last decade (Cipra 1996), it does not meet any of the above asymptotes so that we omit it in this brief review. In addition, for recent progresses on the power law and composite expansions, readers are referred to Barenblatt et al. (1997b), Gorge and Casterlo (1997), Afzal (2001), and Panton (2005). Finally, several high quality experimental studies on the effects of Reynolds number have been reported in the last decade, they are fundamental to turbulence research and will be detailed when used.

The purpose of this paper is to solve the mean flow similarity problem in two steps: First, we construct two compound similarity numbers to collapse mean velocity profile data with different Reynolds numbers to a single universal profile. Then, by applying asymptotic analysis, we propose a self-similarity law that describes the entire mean flow profile and meets all asymptotic requirements.

\section{Hypotheses of self-similarity}

\section{A. Formulation of hypotheses}

We have mentioned that we take Eq. (13) as our preliminary analysis. Recently, we reanalyzed the Zagarola and Smits superpipe data set and found that if the number 3 in the correction term is changed to be 4, Eq. (13) fits the experimental data even better. Thus, we rewrite Eq. (13) as

$$
u^{+}=\left(\frac{1}{\kappa} \ln y^{+}+B\right)+\frac{2 \Pi}{\kappa} \sin ^{2} \frac{\pi \xi}{2}-\frac{\xi^{4}}{4 \kappa}
$$

By considering $\Pi \approx \kappa$ (Guo and Julien 2003), we can rearrange the equation above as

$$
u^{+}-2 \sin ^{2} \frac{\pi \xi}{2}=\frac{1}{\kappa}\left(\ln y^{+}-\frac{\xi^{4}}{4}\right)+B
$$

or

$$
U=\frac{1}{\kappa} \ln Y+B
$$

where

$$
Y \equiv y^{+} \exp \left(-\frac{\xi^{4}}{4}\right)
$$

is a combination of the inner and outer variables, and

$$
U \equiv u^{+}-2 \sin ^{2} \frac{\pi \xi}{2}
$$

is the pure effect of the wall. We call $Y$ and $U$ two compound similarity numbers that switch to the inner variables $y^{+}$and $u^{+}$, respectively, when $\xi \rightarrow 0$; we call Eq. (14c) the general log law that is expected to be valid in the whole outer region and reduces to the classic log law in the overlap where $\xi \rightarrow 0$.

Our hypotheses are based on the analysis of Eqs. (14a)-(14e).

Hypothesis I: For large Reynolds number turbulence, say Re $>10^{5}$, a pure outer region exists. The mean flow in the outer region can be described with the general $\log$ law of eq.(14c), where $\kappa$ and $B$ are universal constants determined experimentally.

Prediction I: We can test Hypothesis I in two ways: (a) if it is true, then the plot of $\gamma=Y d U / d Y$ versus $Y$ for different Reynolds numbers must be a universal constant $1 / \kappa$, i.e.

$$
\gamma=Y \frac{d U}{d Y}=\frac{1}{\kappa}=\text { const }
$$


where the value of $\kappa$ is determined by Eq. (10), and $\gamma$ is called a diagnostic function for the log law according to Österlund et al. (2000); (b) if it is true, then experimental data with different Reynolds numbers in the outer region must collapse to a single straight line in coordinates $(\ln Y, U)$.

Hypothesis II: In the inner region where $\xi \rightarrow 0$, the two similarity numbers become $Y \rightarrow y^{+}$and $U \rightarrow u^{+}$, which is the law of the wall, Eq. (3). We then hypothesize that the two similarity numbers can also collapse experimental data in the inner region to a single profile. Consequently, the two similarity numbers are expected to describe the entire velocity profile from the wall to axis.

Prediction II: If Hypothesis II is true, experimental profiles with different Reynolds numbers must fall into a single curve for the entire flow domain.

Hypothesis III: For moderate Reynolds number, say $10^{4} \leq \mathrm{Re}<10^{5}$, the effect of viscosity may penetrate till the axis. In such a case, a pure outer region may not exist at all. But in terms of $(Y, U)$, the flow near the pipe axis might be similar to that near the wall in large Reynolds number turbulence.

Prediction III: If Hypothesis III is true, then experimental profiles with moderate Reynolds numbers must superpose with the near wall data of large Reynolds number flows.

We exclude the low Reynolds number effect, say $\operatorname{Re}<10^{4}$, in the above hypotheses.

\section{B. Test of the hypotheses}

Data sets: The test of the above three hypotheses needs high quality experimental data sets, which have been available in a comprehensive report by the Working Group 21 of the Fluid Dynamics Panel of AGARD (1997). Five pipe data sets are compiled in Chapter 5 of the report. They are PCH00 by Loulou et al. (1997a, b) that is a DNS set and has been used in determining the asymptote of Eq. (7) in Fig. 1, PCH01 by Durst et al. $(1995,1997)$, PCH02 by Perry et al. $(1986,1997)$, PCH03 by den Toonder and Nieuwstadt (1997a, b), and PCH04 by Zagarola and Smits $(1997,1998)$. Besides, we also use the recent Princeton superpipe data set by McKeon et al. (2004) for this evaluation. The flow characteristics for each set are described when used.

Determination of the log law constants: The general and classic log laws have the same constants. Let us determine the values of $\kappa$ and $B$ by using the Milikan asymptote of Eq. (10). This method makes no assumptions as to the nature of turbulent motions. We plot the maximum velocities of the Princeton data sets (Zagarola and Smits 1997, McKeon et al. 2004) in Fig. 2, according to Eq. (10). The Zagarola and Smits set gives $\left(\kappa, B_{1}\right)=(0.44,7.86)$ while the McKeon et al. set leads to $\left(\kappa, B_{1}\right)=(0.43,7.30)$. Since the Zagarola and Smits set is not corrected for the Pitot probe displacement (McKeon et al. 2003) while the set of McKeon et al. is fully corrected for effects of viscosity, turbulence, velocity gradient and the presence of a wall, we take the later for the standard values of $\kappa$ and $B_{1}$ in this paper, i.e.

$$
\kappa=0.43, \quad B_{1}=7.3
$$

On the other hand, we can find the maximum velocity with Eq. (14a),

$$
u_{\max }^{+}=\frac{1}{\kappa} \ln \mathrm{Re}_{*}+\left(B+2-\frac{1}{4 \kappa}\right)
$$

Comparing it with Eq. (10) gives

$$
B_{1}=B+2-\frac{1}{4 \kappa}
$$

Substituting the values of Eq. (16) gives

$$
B=5.9 \approx 6
$$

With the constants in Eqs. (16) and (18), we plot the maximum velocity of Eq. (10) and the classic log law of Eq. (9) in Figs. 4a, 5a, 6a and 7a. Except for Fig. 7a, good agreement between the log laws and the data can be observed. In particular, the von Kármán constant $\kappa=0.43$ works excellently for the envelope in Fig. $6 \mathrm{a}$, which is from a different facility from the Princeton sets. For Fig. 7a, we notice that the classic log law fails to describe this data set since it cannot unify the data beyond the buffer layer, with different Re, to a single straight line. We will further discuss it in the following sections.

Test I: This test corresponds to Hypothesis I and Prediction I where Reynolds number is large. The two Princeton superpipe data sets (Zagarola and Smits 1997, McKeon et al. 2004) and the Perry et al. (1986, 1997) set are suitable to this test. In the Princeton sets, the velocities were measured with Pitot probes; the 

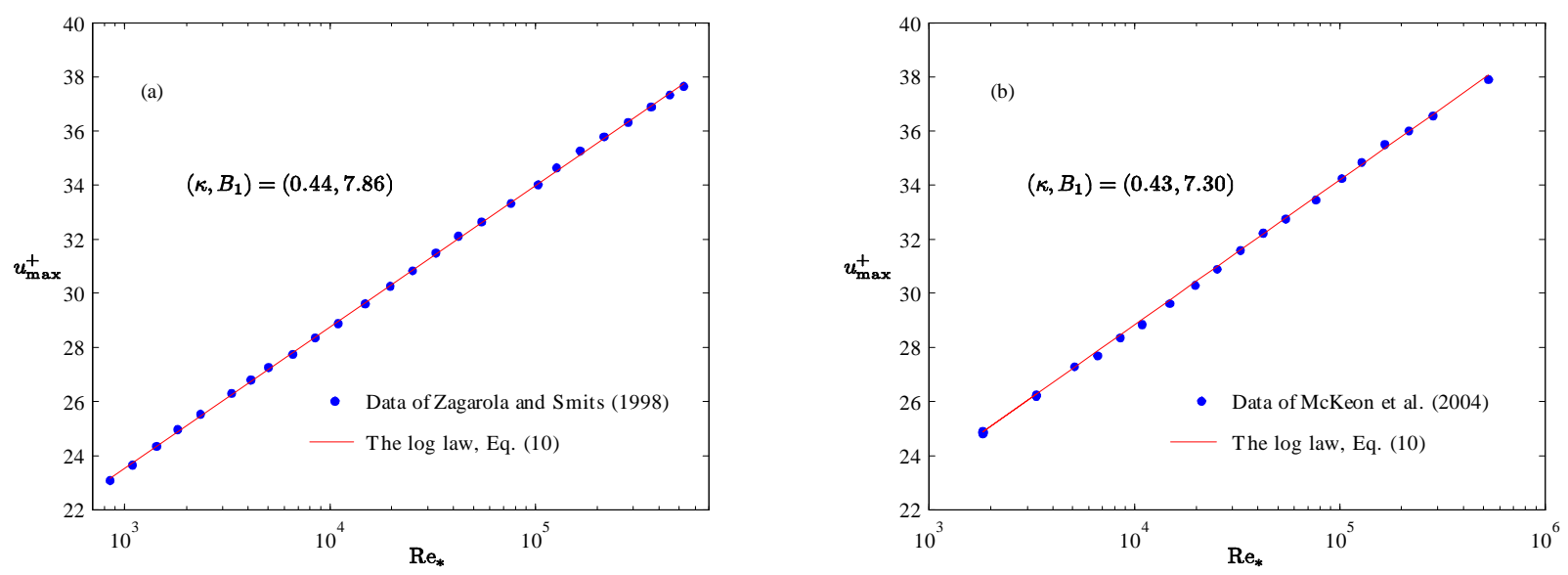

Figure 2. Determination of the von Karman constant by the maximum velocity: (a) the Zagarola and Smits data set; (b) the McKeon et al. data set.

bulk Reynolds number Re is between $31 \times 10^{3}$ and $36 \times 10^{6}$; and the Kármán number is between 1700 and $530 \times 10^{3}$. The detailed information can be found in Zagarola (1996) who surveyed 26 mean velocity profiles. Although the profiles lack the displacement correction, they do not much affect the qualitative evaluation of the hypotheses. From the same facility, McKeon et al. (2004) repeated the measurements by Zagarola and further measured 25 velocity profiles that are fully corrected for the effects of viscosity, turbulence, velocity gradient and the presence of the wall. In the Perry set, the velocities were also measured with Pitot probes, $\operatorname{Re}=(75-200) \times 10^{3}$ and $\operatorname{Re}_{*}=1586-3838$.

We first plot the superpipe data in Fig. 3 according to the diagnostic function of Eq. (15). It shows that the value of $\gamma$ is indeed the constant $\gamma=1 / \kappa=1 / 0.43=2.33$ when $Y \geq 1000$, which is consistent with our Prediction I. This also implies that we can define the outer region as $Y \geq 1000$. We then plot the three data sets in Figs. 4, 5 and 6, respectively, in both the conventional inner coordinates $\left(\ln y^{+}, u^{+}\right)$and our compound similarity coordinates $(\ln Y, U)$. As aforementioned, there exists a log layer in Figs. 4a, 5a and 6a, but the inner coordinates cannot collapse all the outer region data to a single curve for different Reynolds numbers. Nevertheless, in Figs. 4b, 5b and $6 \mathrm{~b}$, all the data in the outer region, say at least $Y \geq 1000$, collapse to a single straight line. This is in full agreement with our Prediction I so that Hypothesis I is confirmed experimentally. In addition, we plot the general log law of Eq. (14c) in Figs. 4b, 5b and 6b, which show excellent agreement although the value of $B=6$ slightly overestimates the Perry data set. We found that the Perry data set corresponds to a value of $B=5.6$. This slight difference might be caused by the wall roughness or different Pitot probe correction in the Perry set.

Test II: Hypothesis II and Prediction II can also be tested with Figs. 4b, 5b and $6 \mathrm{~b}$ where both the conventional inner coordinates and our compound similarity coordinates can collapse the inner region data to a single profile. Nevertheless, only the compound similarity coordinates $(Y, U)$ can unify the two regions to a single curve, as expected in Prediction II.

Test III: This test checks Hypothesis III and Prediction III for moderate Reynolds numbers where Re is in the order of $10^{4}$. In fact, Figs. 4, 5 and 6 have included a few of profiles with moderate Re. With LDA technique, den Toonder and Nieuwstadt $(1997 \mathrm{a}, \mathrm{b})$ measured four velocity profiles with $\mathrm{Re}=4900-24580$ or $\mathrm{Re}_{*}=328-1380$. This set has been used to compare the buffer layer measurements and calibrate Pitot probes (Zagarola et al. 1997, McKeon et al. 2003). We plot all the four profiles in the conventional inner coordinates $\left(\ln y^{+}, u^{+}\right)$in Fig. 7a and the three moderate Re profiles in our compound similarity coordinates $(\ln Y, U)$ in Fig. 7b, respectively. Figure 7a shows that a pure log law layer does not exist for this set due to the small Reynolds numbers, as stated in Hypothesis III. However, in Fig. 7b, our similarity numbers can well superpose the measured data with different moderate Re to a single curve. To check if the data of moderate Re superpose with the near wall data of large Re, we superpose Figs. 5b, 6b and $7 \mathrm{~b}$ to produce Fig. 8. As expected in Prediction III, the moderate Re data coincide with the lower part of large Re data. Note that: (1) we exclude the Zagarola and Smits set in Fig. 8 since its uncorrected data in the buffer layer have large errors; and (2) we cannot estimate the von Kármán constant with the den Toonder set since the 


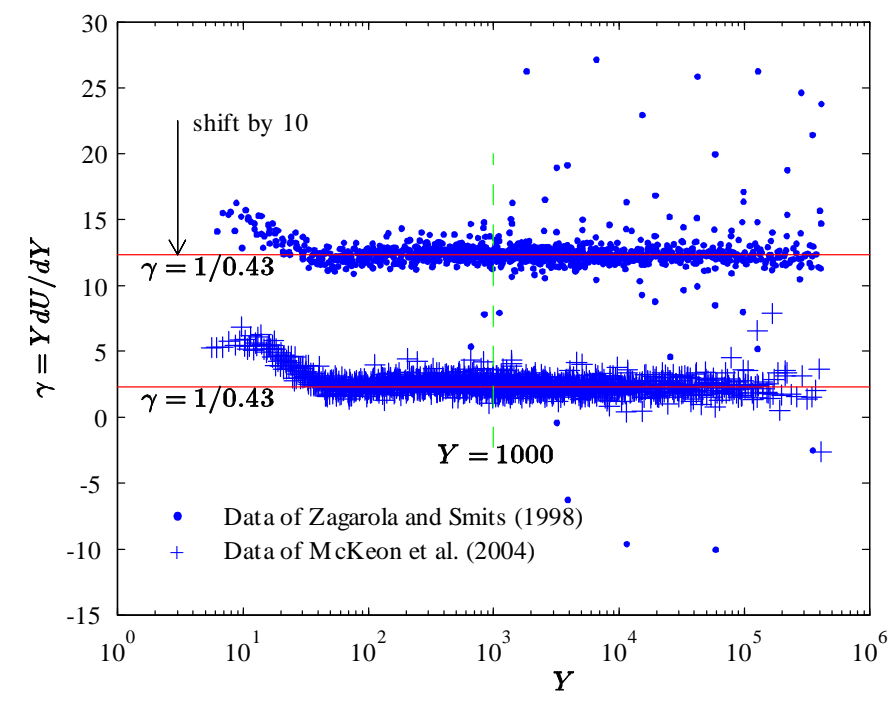

Figure 3. Test of Hypothesis I, the general log law, with the diagnostic function $\gamma$
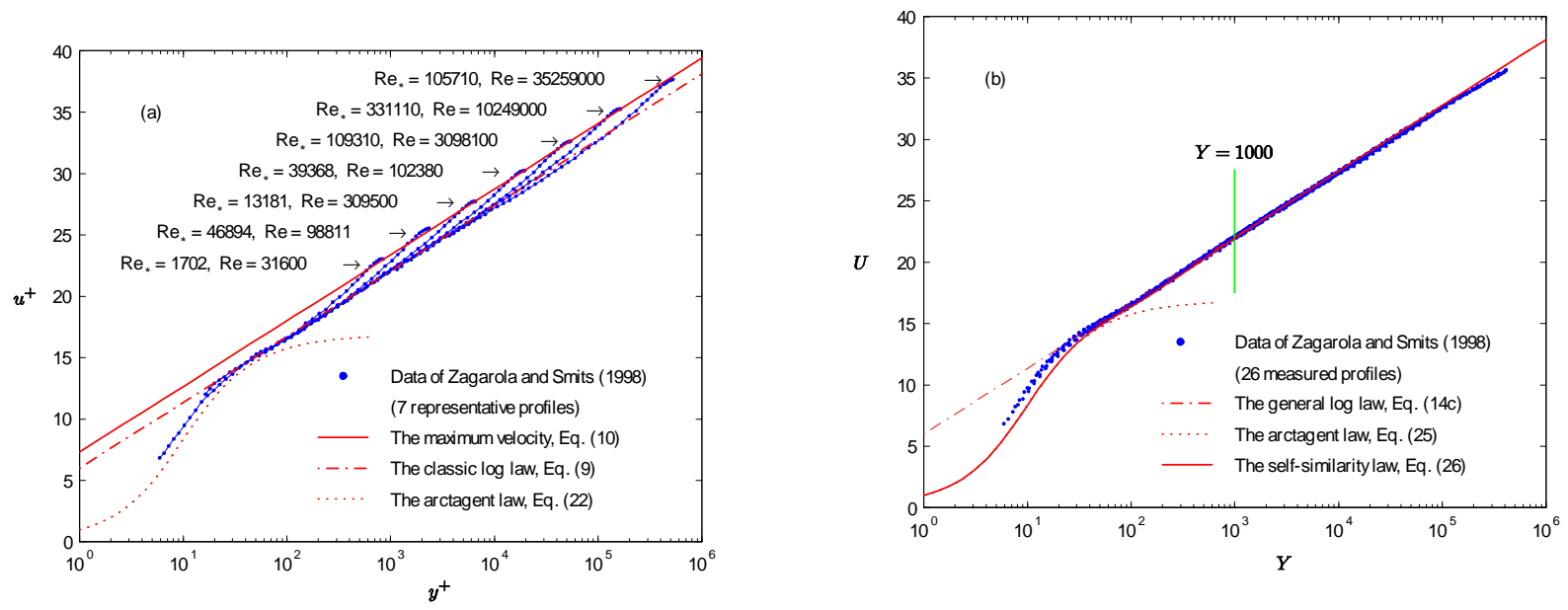

Figure 4. Test of the compound similarity hypothesis with data of Zagarola and Smits: (a) representative velocity profiles with different Reynolds numbers in wall coordinates $\left(y^{+}, u^{+}\right)$; (b) 26 measured velocity profiles in compound similarity coordinates $(Y, U)$. This data set inspired the generation of the compound similarity hypothesis. 

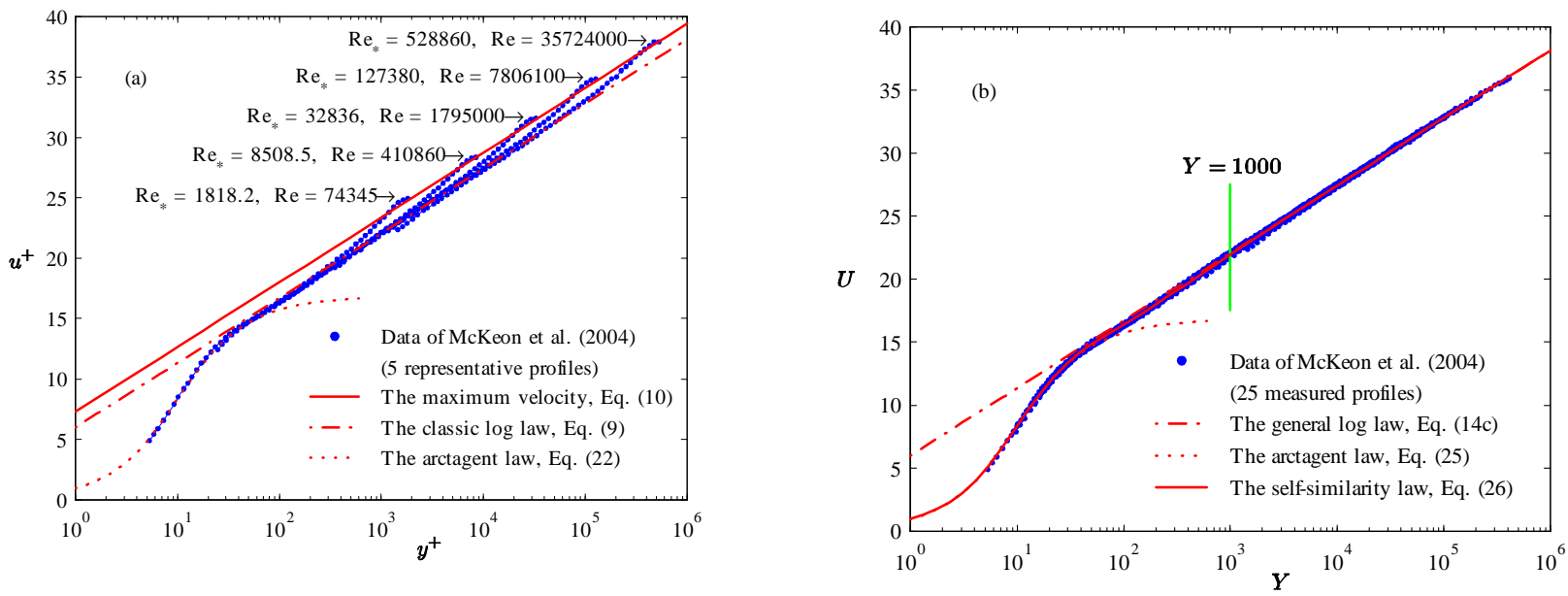

Figure 5. Test of the compound similarity hypothesis with data of McKeon et al.: (a) representative velocity profiles with different Reynolds numbers in wall coordinates $\left(y^{+}, u^{+}\right)$; (b) 25 measured velocity profiles in compound similarity coordinates $(Y, U)$.
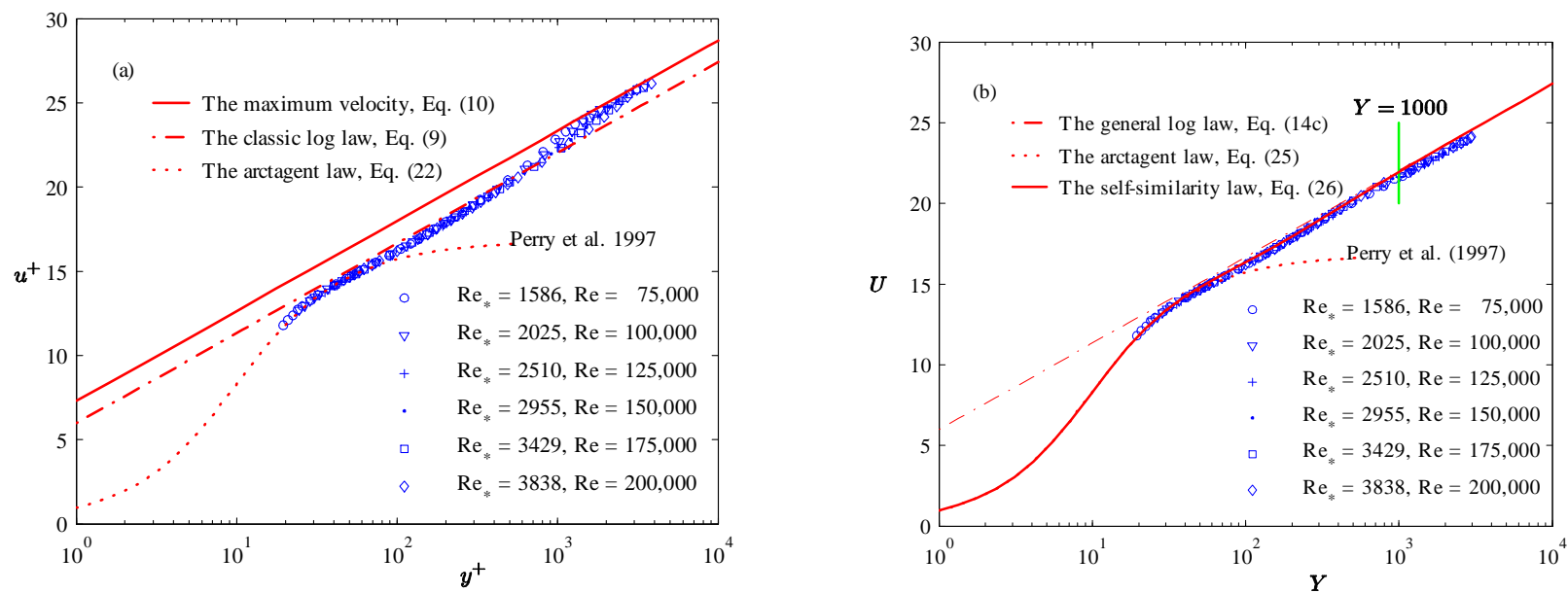

Figure 6. Test of the compound similarity hypothesis with data of Perry et al.: (a) in wall coordinates $\left(y^{+}, u^{+}\right)$; (b) in compound similarity coordinates $(Y, U)$. 

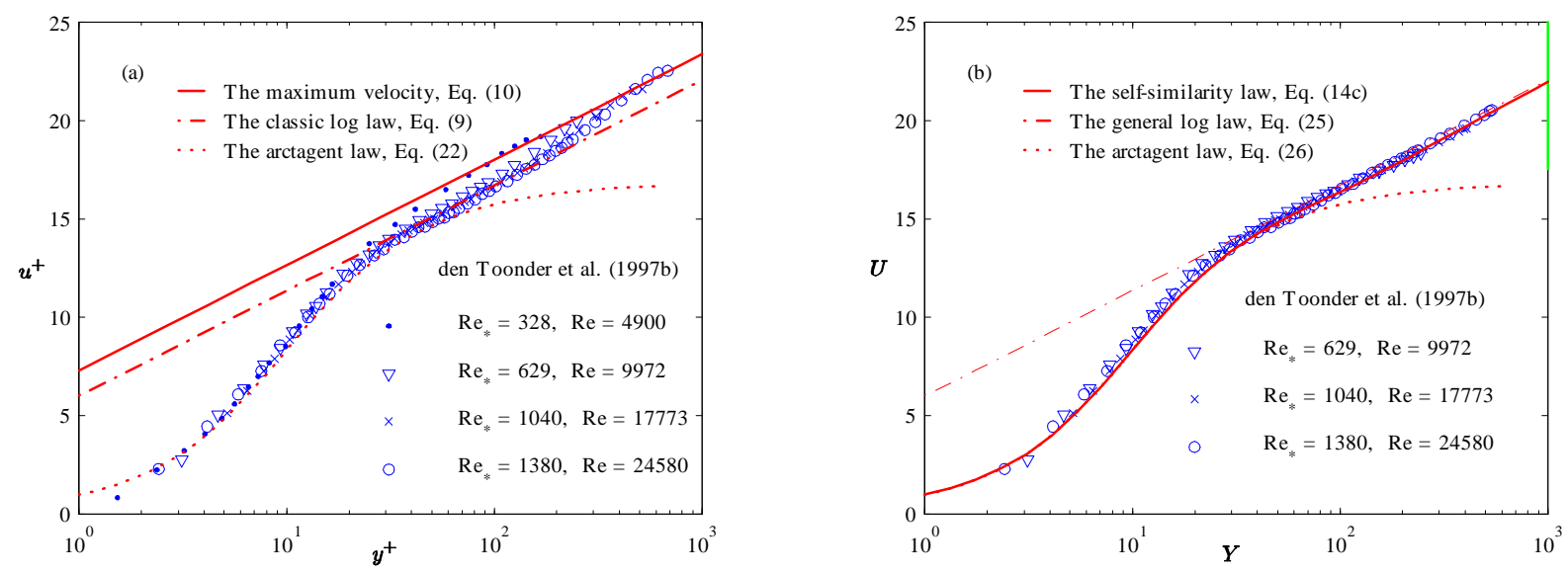

Figure 7. Test of the compound similarity hypothesis with data of den Toonder and Nieuwstadt: (a) in wall coordinates $\left(y^{+}, u^{+}\right) ;($b) in compound similarity coordinates $(Y, U)$.

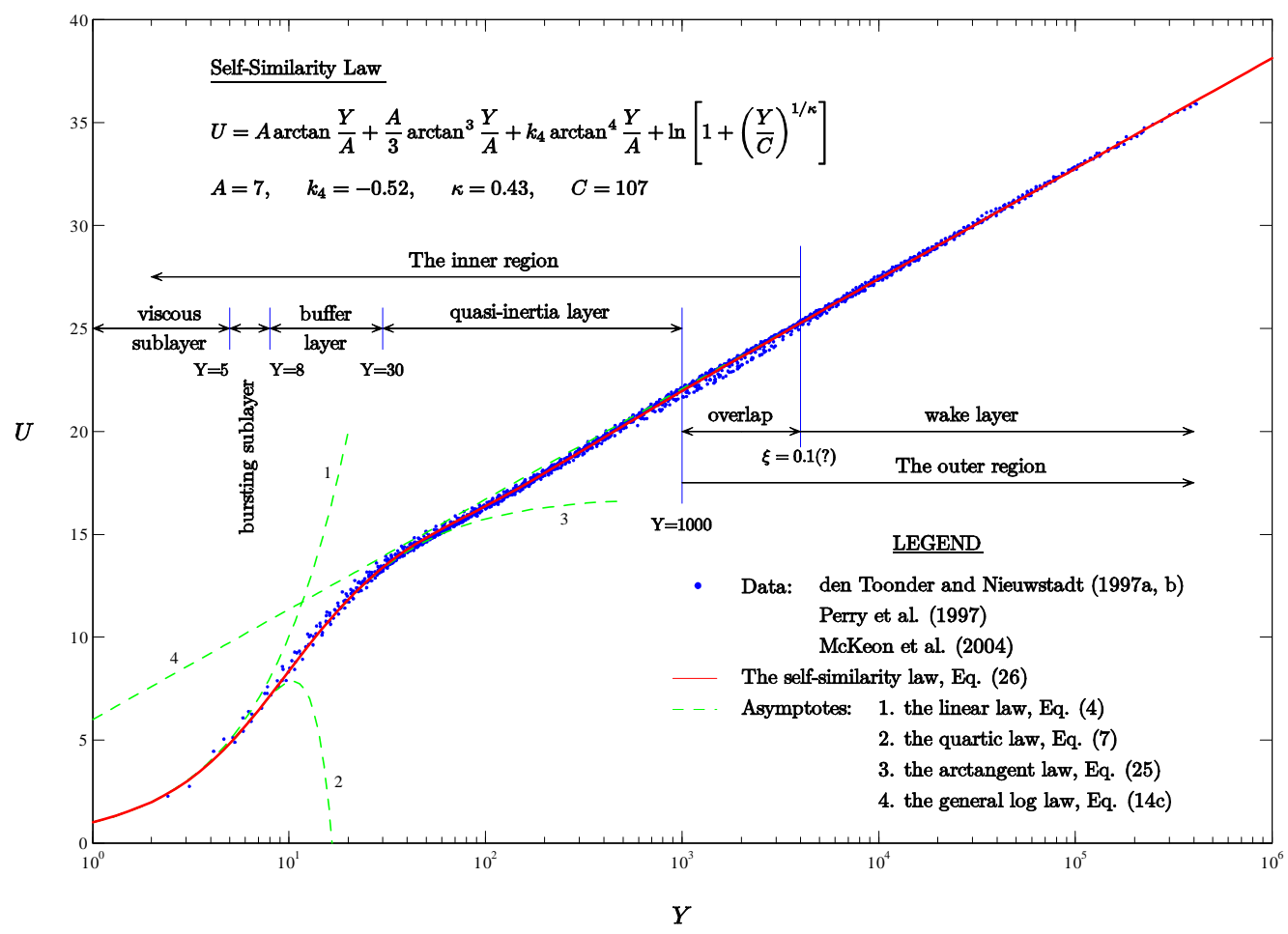

Figure 8. Check of the universality of the self-similarity law with both large and moderate Reynolds numbers 
prerequisite, $\operatorname{Re}_{*} \rightarrow \infty$, does not exist. To distinguish such a case from the real outer region, we propose a quasi-inertia layer between the buffer and overlap layers, shown in Fig. 8.

In summary, we have answered the question raised in this paper. That is, our two compound similarity numbers $(Y, U)$ can well collapse experimental mean velocity profiles with different moderate and large Reynolds numbers to a single universal profile. In other words, a self-similarity exists in terms of $(Y, U)$ for the mean flows with different Reynolds numbers.

\section{Self-similarity law}

\section{A. The arctangent law in the buffer layer}

To model the universal velocity profile in Fig. 8, we start with the buffer layer, which is defined between $y^{+}=8$ and 30. We consider the quasi-inertia layer a transition from the buffer to the inertia (overlap) layer; and the buffer layer connects the inertia layer through the additive constant $B$. We then conjecture that the buffer layer model must tend to a constant when $y^{+} \rightarrow \infty$. The arctangent function has such characteristics so that we assume the velocity profile in the buffer layer can be approximated with a series of arctangent functions,

$$
u^{+}=\sum_{i=1}^{\infty} k_{i} \arctan ^{i} \frac{y^{+}}{A}
$$

where $A$ is a characteristic value of $y^{+}$in the bursting sublayer. The values of $A$ and coefficients $k_{i}$ are determined with the asymptote of Eq. (7) and experimental data.

Since the secondary term in Eq. (7) is quartic, let us expand Eq. (19) to the forth power, i.e.

$$
\begin{aligned}
u^{+} & =k_{1} \arctan \frac{y^{+}}{A}+k_{2} \arctan ^{2} \frac{y^{+}}{A}+k_{3} \arctan ^{3} \frac{y^{+}}{A}+k_{4} \arctan ^{4} \frac{y^{+}}{A}+\cdots \\
& =\frac{k_{1}}{A} y^{+}+\frac{k_{2}}{A^{2}} y^{+2}+\left(\frac{k_{3}}{A^{3}}-\frac{k_{1}}{3 A^{3}}\right) y^{+3}+\left(\frac{k_{4}}{A^{4}}-\frac{2 k_{2}}{3 A^{4}}\right) y^{+4}+\cdots
\end{aligned}
$$

Comparing it with Eq. (7) gives

$$
k_{1}=A, k_{2}=0, k_{3}=\frac{A}{3}, k_{4}=-\frac{\lambda A^{4}}{4}=-\frac{A^{4}}{4600}
$$

where Eq. (8) has been used. This means we can approximate Eq. (19) as

$$
u^{+}=A \arctan \frac{y^{+}}{A}+\frac{A}{3} \arctan ^{3} \frac{y^{+}}{A}+k_{4} \arctan ^{4} \frac{y^{+}}{A}
$$

By applying the data set of Durst et al. $(1995,1997)$ where the Reynolds numbers are relatively low and the buffer layers are amplified in the LDA measurements, we fit the value of $A$ to be

$$
A=7
$$

and from Eq. (21) we get

$$
k_{4}=-0.52
$$

Figure 9 reveals that the arctangent law of Eq. (22) agrees very well with the data till $y^{+}=30$. Note that this set was not used in the test of the hypotheses because fully developed flows near the axis are not well established, as described by the authors (Durst et al. 1997). Equation (22) is also supported by Figs. 5a, 6a

and 7a. No surprising, the data in Fig. 4a deviate from the arctangent law considerably, due to the lack of displacement correction of the Pitot probe. As an application of this study, we will correct this data set later in this paper. Besides, we did not plot Eq. (22) in Fig. 1 where the Re is too low to have a fully developed buffer layer.

To match the arctangent law of Eq. (22) with the general log law of Eq. (14c), we rewrite Eq. (22) according to Hypothesis II,

$$
U=A \arctan \frac{Y}{A}+\frac{A}{3} \arctan ^{3} \frac{Y}{A}+k_{4} \arctan ^{4} \frac{Y}{A}
$$

which is compared with data in $4 \mathrm{~b}, 5 \mathrm{~b}, 6 \mathrm{~b}, 7 \mathrm{~b}$, and 8 . The agreement is similar to those in Figs. 4a, 5a, 6a and $7 \mathrm{a}$. 


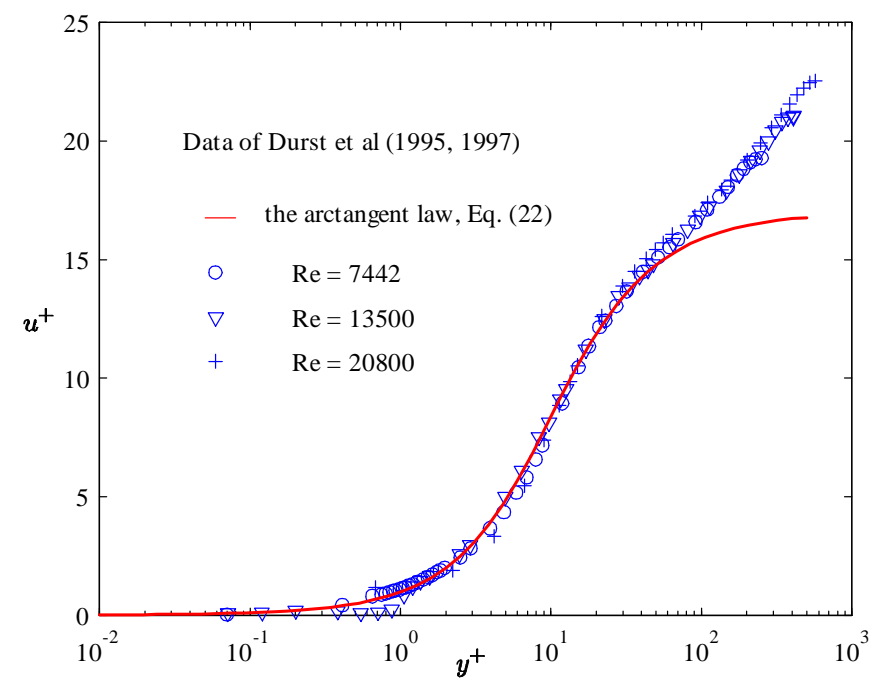

Figure 9. Check of the arctangent law in the buffer layer

\section{B. The self-similarity law}

A complete self-similarity law can be constructed by matching the arctangent law of Eq. (25) with the general log law of Eq. (14c),

$$
U=A \arctan \frac{Y}{A}+\frac{A}{3} \arctan ^{3} \frac{Y}{A}+k_{4} \arctan ^{4} \frac{Y}{A}+\ln \left[1+\left(\frac{Y}{C}\right)^{1 / \kappa}\right]
$$

where the values of $\kappa, A$ and $k_{4}$ are specified with Eqs. (16), (23) and (24). The value of $C$ is determined by letting $Y \rightarrow \infty$, i.e.

$$
U=\underbrace{\frac{\pi A}{2}+\frac{A}{3}\left(\frac{\pi}{2}\right)^{3}+k_{4}\left(\frac{\pi}{2}\right)^{4}-\frac{1}{\kappa} \ln C}_{B}+\frac{1}{\kappa} \ln Y
$$

Comparing it with the general log law of Eq. (14c) gives

$$
C=\exp \left[\kappa\left(\frac{\pi A}{2}+\frac{A}{3}\left(\frac{\pi}{2}\right)^{3}+k_{4}\left(\frac{\pi}{2}\right)^{4}-B\right)\right]
$$

Substituting the values of $\kappa, A, k_{4}$ and $B=6$ gives

$$
C=107
$$

Figure 8 compares the self-similairity law, Eq. (26), with the data sets of McKeon et al. (2004), Perry et al. (1997) and den Toonder and Nieuwstadt (1997a, b). To minimize the data band, all data are denoted with the symbol of dot. The figure shows that the self-similarity law can really clone the experimental profiles very well from the wall to axis.

In summary, we have found two compound similarity numbers $Y$ and $U$ that can collapse experimental velocity profiles with different Reynolds numbers to a single profile; the empirical self-similarity law of Eq. (26) embeds all asymptotic requirements and the sine-square wake law and can describe the entire velocity profile from the wall to axis.

\section{Application: Displacement correction for Zagarola and Smits superpipe data set}

The Zagarola and Smits $(1997,1998)$ superpipe data have been widely cited in literature (Barenblatt 1997a, Perry et al. 2001, Guo and Julien 2003, and many others). However, the uncorrected data due to the 


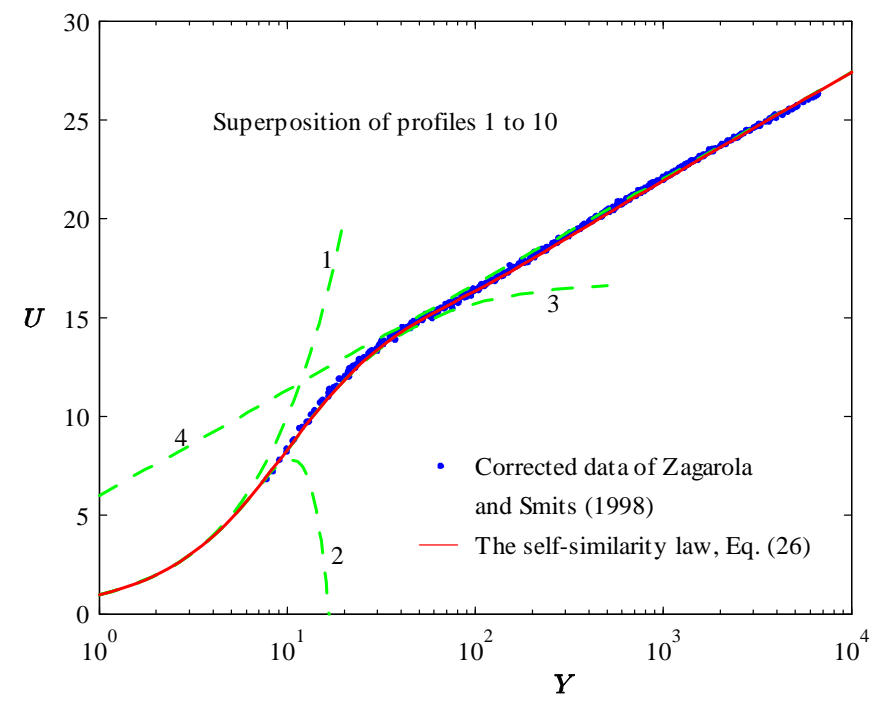

Figure 10. Comparison of the corrected data of Zagarola and Smits with the self-similarity law

velocity gradient or streamline displacement caused large errors in the buffer layer. McKeon et al. (2003) proposed the following equation for the Pitot probe correction:

$$
\frac{\Delta y}{D_{p}}=0.15 \tanh (4 \sqrt{\alpha})
$$

where $\Delta y=$ correction in position due to streamline displacement, $D_{p}=$ diameter of Pitot probe, and $\alpha=$ Dimensionless velocity gradient defined by

$$
\alpha=\left.\frac{1}{2} \frac{D_{p}}{u\left(y_{c}\right)} \frac{d u}{d y}\right|_{y=y_{c}}
$$

where $u\left(y_{c}\right)=$ true velocity at the center of the probe $y=y_{c}$. To apply the above equations, we must know the accurate values of the velocity and velocity gradient at the center of the probe, which can now be found by the arctangent law of Eq. (22) for the buffer layer or the self-similarity law of Eq. (26) for the entire profile. Figure 10 shows the self-similarity law with the corrected data for the first 10 profiles where there are measurement data in the buffer layers. Since the self-similarity law has been verified in the last section, we believe the correction is a good representation of the true measured data.

\section{Summary and Conclusions}

From the preliminary analysis of the modified log-wake law, Eqs. (13) and (14a), we have proposed two compound similarity numbers $(Y, U)$ to describe the pipe mean velocity profiles with different Reynolds numbers. As shown in Eqs. (14d) and (14e), the number $Y$ expresses a combination of the inner variable $y^{+}=y u_{*} / \nu$ and outer variable $\xi=y / R$, and the number $U$ is the pure effect of the wall where the effect of the pressure-gradient has been subtracted.

With the two compound similarity numbers, we made three hypotheses for large and moderate Re turbulence: i) for large Re, the outer region can be described with the general log law of Eq. (14c); ii) for large Re, the two similarity numbers can also collapse the inner region data to a single profile; and iii) in terms of $(Y, U)$, there is a similarity between moderate and large Re turbulence.

These hypotheses have been confirmed with experimental data. i) The Princeton superpipe data sets and the Perry et al. experiments confirmed that the outer region flow, where $Y \geq 1000$, indeed follows the general $\log$ law of Eq. (14c), as shown in Figs. 4b, 5b and 6b. The von Kármán constant and the additive constant are determined to be $(\kappa, B)=(0.43,6)$ with Milikan's maximum velocity equation of Eq. (10) and 
the McKeon et al. superpipe data set. ii) Figures $4 \mathrm{~b}, 5 \mathrm{~b}$ and $6 \mathrm{~b}$ also confirmed that the similarity numbers $(Y, U)$ can collapse the inner flow data to a single profile, but a quasi-inertia layer exists between the buffer layer and logarithmic overlap. iii) The den Toonder and Nieuwstadt data set in Figs. 7b and 8 confirmed that the two compound similarity numbers can superpose moderate Re data with those of large Re. In general, the two numbers can collapse large and moderate Re mean velocity profile data to a single universal profile.

To model the buffer layer velocity profile, we proposed an arctangent law of Eq. (22) or (25) that compares with the data in Figs. 4, 5, 6, 7, 8 and 9. Matching the arctangent law and the general log law, we obtained the self-similarity law of Eq. (26). Figure 8 confirms that the self-similarity law can describe the entire velocity profile from the wall to axis and satisfies all asymptotic requirements specified in Eqs. (4), (7), (9) and (12) as well as the widely accepted since-square wake law.

As an application of the proposed laws, we improved the McKeon displacement correction for Pitot probe data, which can be used to correct the widely used Zagarola and Smits superpipe data set. Finally, we expect that the two compound similarity numbers and the self-similarity law can be extended to channel flows and turbulent boundary layers.

\section{Acknowledgments}

The author thanks Dr. P. Y. Julien with Colorado State University for his encouragement during this study. The author is also grateful to Dr. M. Zagarola with CREARE Engineering Research and Development, Dr. M. Chong with the University of Melbourne in Australia, Dr. J. M. J. den Toonder with Philips Research Laboratories in the Netherlands, and Dr. M. H. Buschmann with Institut für Luft- und Kältetechnik Dresden in Germany for their comments and providing their data sets. The research was sponsored by the Academic Research Grant of the University of Nebraska - Lincoln under grant No. 27-1120-0005-002.

\section{References}

Afzal, N. (2001). "Power law and log law velocity profiles in fully developed turbulent pipe flow: equivalent relations at large Reynolds numbers." Acta Mechanica, 151, 171-183.

Barenblatt, G. I. (1993). "Scaling laws for fully developed turbulent shear flows. Part I: Basic hypothesis and analysis." J. Fluid Mech. 248, 513-520.

Barenblatt, G. I., Chorin, A. J. and Prostokishin V. M. (1997a). "Scaling laws for fully developed turbulent flow in pipes: Discussion of experimental data." Proc. Natl. Acad. Sci., 94, 773-776.

Barenblatt, G. I. Chorin, A. J. and Prostokishin, V. M. (1997b). "Scaling laws in fully developed turbulent pipe flow." Appl. Mech. Rev. 50, 413-429.

Buschmann, M. H. and Gad-el-Hak, M. (2005). "New mixing-length approach for the mean velocity profile of turbulent boundary layers." J. Fluids Engrg., ASME, 127(3), 393-396.

Chapmann, D. R. and Kuhn, G. D. (1986). "The limiting behaviour of turbulence near a wall." J. Fluid Mech., 170, 265-292.

Cipra, B. (1996). "A new theory of turbulence causes a stir among experts." Science, 272(5264), 951.

Coles, D. E. (1956). "The law of the wake in the turbulent boundary layer." J. Fluid Mech., 1, 191-226.

den Toonder, J. M. J. and Nieuwstadt, F. T. M. (1997a). "Reynolds number effects in a turbulent pipe flow for low to moderate Re." Phys. Fluids, 9(11), 3398-3409.

den Toonder, J. M. J. and Nieuwstadt, F. T. M. (1997b). "PCH03: Turbulent pipe flow experiment." http://torroja.dmt.upm.es/ftp/AGARD/chapter5/.

Durst, F., Jovanovic, J. and Sender, J. (1995). "LDA measurements in the near-wall region of a turbulent pipe flow." J. Fluid Mech., 295, 305-335. 
Durst, F., Jovanovic, J. and Sender, J. (1997). "PCH01: Turbulent pipe flow experiments." http://torroja.dmt.upm.es/ftp/AGARD/chapter5/.

Gad-el-Hak, M. (1997). "Editorial: The last conundrum." Appllied Mathematics, 50(12), 1-6.

George, W. K. and Castillo, L. (1997). "The zero-pressure-gradient turbulent boundary layer: An Assessment of the data." Applied Mech. Review, 50(12), Part I, 689-729.

Guo, J., and Julien, P. Y. (2003). "Modified log-wake law for turbulent flow in smooth pipes." J. Hydraul. Res., IAHR, 41(5), 493-501.

Guo, J., Julien, P. Y. and Meroney, R. N. (2005). "Modified log-wake law for zero-pressure-gradient turbulent boundary layers." J. Hydraul. Res., 43(4), 421-430.

Grass, AJ (1971). "Structural features of turbulent flow over smooth and rough boundaries." J. Fluid Mech., 50(2), 233-255.

Grass, A. J. , Stuart, R. J. and Mansour-Tehrani, M. (1991). "Vortical Structures and Coherent Motion in Turbulent Flow over Smooth and Rough Boundaries." Philosophical Transactions: Physical Sciences and Engineering, 336, No. 1640, 35-65.

Hinze, J. O. (1975). Turbulence. 2nd Ed., McGraw-Hill.

Kim, H. T., Kline, S. J., Reynolds, W. C. (1971). "The production of turbulence near a smooth wall in a turbulent boundary layer." J Fluid Mech. 50 (Part 1): 133-160.

Kline, S. J., Reynolds, W. C., Shraub, F. A. and Runstadler, P. W. (1967). "The structure of turbulent boundary layers." J Fluid Mech, 30, 741.

Loulou, P., Moser, R., Mansour, N. and Cantwell, B. (1997a). Direct simulation of incompressible pipe flow using a b-spline spectral method. Technical Report TM 110436, NASA.

Loulou, P., Moser, R., Mansour, N. and Cantwell, B. (1997b). "PCH00: Fully developed turbulent pipe flow simulation." http://torroja.dmt.upm.es/ftp/AGARD/chapter5/.

McKeon, B. J., Li, L., Jiang, W., Morrison, J. F. and Smits, A. J. (2003). "Pitot probe corrections in fully developed turbulent pipe flow." Measurement Science and Technology, 14, 1449-1458.

McKeon, B. J., Li, J., Jiang, W., Morrison, J. F. and Smits, A. J. (2004). "Further observations on the mean velocity distribution in fully developed pipe flow." J. Fluid Mech., 501, 135-147.

Milikan, C. B. A. (1938). "A critical discussion of turbulent flows in channels and circular tubes." Proc. of 5th International Congress on Applied Mechanics, 386-392.

Nelkin, M. (1992). "In what sense is turbulence an unsolved problem?" Science, 255, 566-570.

Österlund, J. M., Johnanson, A. V., Nagib, H. M. and Hites, M. H. (2000). "A note on the overlap region in turbulent boundary layers." Phys. Fluids, 12(1), 1-4.

Panton, R. (2005). "Review of wall turbulence as described by composite expansions." Applied Mechanics Review, 58(1), 1-36.

Perry, A. E., Hafez, S. and Chong, M. S. (2001). "A possible reinterpretation of the Princeton superpipe data." J. Fluid Mech., 439, 395-401.

Perry, A. E., Henbest, S. M. and Chong, M. S. (1986). "A theoretical and experimental study of wall turbulence." J. Fluid Mech., 165, 163-199.

Perry, A. E., Henbest, S. M. and Chong, M. S. (1997). "PCH02: Turbulent pipe flow experiments." http://torroja.dmt.upm.es/ftp/AGARD/chapter5/.

Schlichting, H. (1979). Boundary-Layer Theory. 7th edition, McGraw-Hill. 
White, F. M. (1991). Viscous Fluid Flow. 2nd edition, McGraw-Hill.

Working Group 21 of the Fluid Dynamics Panel of AGARD (1997). http://torroja.dmt.upm.es/ftp/AGARD/.

Zagarola, M. (1996). Mean-flow scaling of turbulent pipe flow. PhD Dissertation, Princeton University, Princeton, NJ.

Zagarola, M. V., Perry, A. E. and Smits, A. J. (1997). "Log laws or power laws: The scaling in the overlap region." Phys. Fluids, 9(7), 2094-2100.

Zagarola, M. V. and Smits, A. J. (1997). "PCH04: Turbulent pipe flow experiments (Superpipe)." http://torroja.dmt.upm.es/ftp/AGARD/chapter5/.

Zagarola, M. V. and Smits, A. J. (1998). "Mean-flow scaling of turbulent pipe flow." J. Fluid Mech., 373, 33-79.

Zanoun, E. Z., Durst, F. and Nagib, H. (2003). "Evaluating the law of the wall in two-dimensional fully developed turbulent channel flows." Phys. Fluids, 15(10), 3079-3088. 